\title{
Collagen sheet dressings for cutaneous lesions of toxic epidermal necrolysis
}

\author{
S. Bhattacharya, H. N. Tripathi' ${ }^{1}$, V. Gupta ${ }^{2}$, Bharti Nigam ${ }^{3}$, A. Khanna ${ }^{4}$ \\ Department of Plastic Surgery, Sahara Hospital and Ajanta Hospital, ${ }^{1}$ Departments of Internal Medicine, ${ }^{2}$ Dermatology and \\ ${ }^{3}$ Ophthalmology, Sahara Hospital, ${ }^{4}$ Department of Surgery, Ajanta Hospital, Lucknow, Uttar Pradesh, India
}

Address for correspondence: Dr. S. Bhattacharya, 907 Mahanagar Setor B, Lucknow 226006, India.

\section{ABSTRACT}

Toxic epidermal necrolysis (TEN) is associated with a significant mortality of $30-50 \%$ and longterm sequelae. Treatment includes early admission to a burn unit, where management with precise fluid, electrolyte, protein, and energy supplementation, moderate mechanical ventilation, and expert wound care can be provided. Specific treatment with immunosuppressive drugs or immunoglobulins did not show an improved outcome in most studies and remains controversial. We have treated the cutaneous lesions of seven patients of TEN with collagen sheet dressings and have found a significant reduction in morbidity. The sheets are a one-time dressing, easy to apply and they reduce fluid loss, prevent infection, reduce pain, avoid repeated dressings and gradually peal off as the underlying lesions heal.

\section{KEY WORDS}

Toxic epidermal necrolysis, Stevens Johnson's Syndrome, collagen sheet

\section{INTRODUCTION}

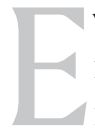

ven though the mechanism of toxic epidermal necrolysis (TEN) is not completely understood, immunologic mechanisms, cytotoxic reactions, and delayed hypersensitivity seem to be involved. Typically, the disease process begins with a nonspecific upper respiratory tract infection. This usually is part of a 1- to 14-day prodrome during which fever, sore throat, chills, headache, and malaise may be present. Vomiting and diarrhoea are occasionally accompany as part of the prodrome. Mucocutaneous lesions develop abruptly.

\begin{tabular}{|l|l|}
\hline \multicolumn{2}{|c|}{ Access this article online } \\
\hline Quick Response Code: & Website: \\
\hline & www.ijps.org \\
\cline { 2 - 2 } & Dol: \\
\hline
\end{tabular}

Clusters of outbreaks last from 2 to 4 weeks. The lesions are typically nonpruritic. The rash can begin as macules that develop into papules, vesicles, bullae, urticarial plaques, or confluent erythema. The centre of these lesions may be vesicular, purpuric, or necrotic.

The typical lesion has the appearance of a target. The target is considered pathognomonic. However, in contrast to the typical erythema multiforme lesions, these lesions have only two zones of colour. The core may be vesicular, purpuric, or necrotic; that zone is surrounded by macular erythema. Some have called these targetoid lesions. Lesions may become bullous and later rupture, leaving denuded skin. The skin becomes susceptible to secondary infection, which in turn is responsible for the scarring associated with morbidity. Although lesions may occur anywhere, the palms, soles, dorsum of the hands, and extensor surfaces are most commonly affected. Mucosal involvement in the form of erythema, oedema, sloughing, 
blistering, ulceration, and necrosis is seen in the oral cavity, conjunctiva, urethra, vagina, nasal vestibule, tracheo-broncheal tree, gastrointestinal tract and anal canal. Consequently, stomatitis, conjunctivitis, urethritis, proctitis, vaginitis, tracheo-bronchitis, pneumonia and enteritis can all occur, complicating the clinical picture.

\section{MATERIALS AND METHODS}

In our unit, in the last 2 years, we have treated the cutaneous lesions of eight patients of TEN with collagen sheet dressings and found a significant reduction in morbidity. Before applying collagen dressing, the affected area is thoroughly cleaned for removal of external contamination, and the infected wound is debrided properly. Collagen sheets are rinsed in normal saline before application. Sheets are applied firmly so as to cover all the cutaneous raw areas. Care is taken to remove air bubbles under the collagen sheet. Subsequently, they can be either dried using a blower or covered by gamjee dressings for the next 72 hours. After removal of the dressing, the collagen sheet stays stuck to the wound and gradually peals off as the wound heals [Figures 1 and 2].

\section{RESULTS AND DISCUSSION}

Biological dressings like collagen are impermeable to bacteria and create the most physiological interface between the wound surface and the environment. Collagen dressings have other advantages over conventional dressings in terms of ease of application and being natural, non-immunogenic, non-pyrogenic,

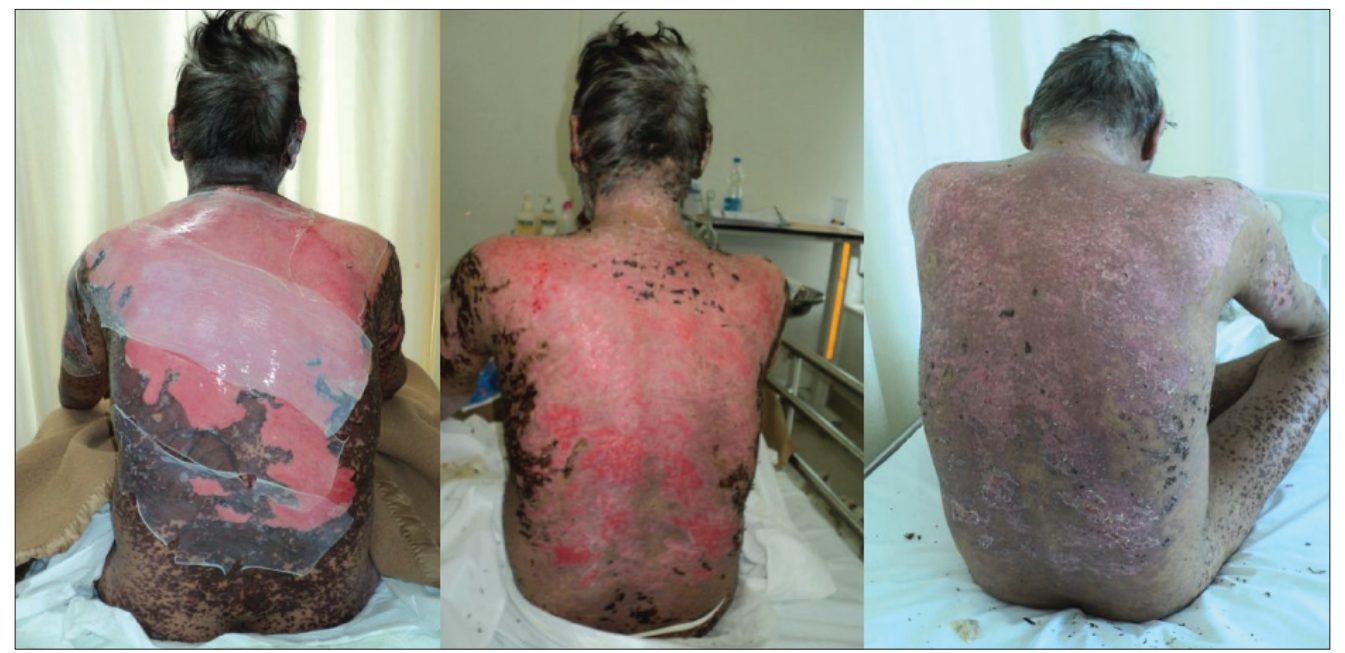

Figure 1: (a) The entire back is involved and covered with collagen sheet dressings; (b) healing is complete and collagen has pealed off on day 10; (c) at the time of discharge on day 15

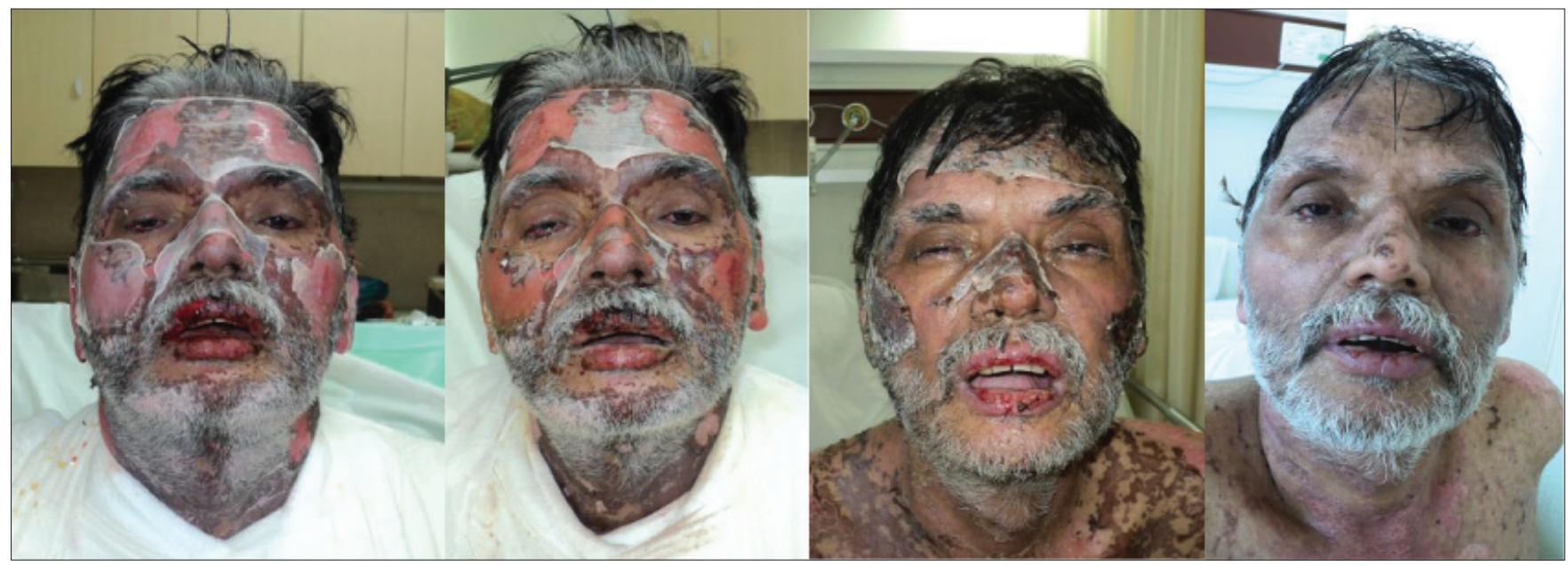

Figure 2: (a) The face dressed with tailored pieces of collagen sheet on day 1 . See the gradual peeling of the sheet as the healing progresses on (b) day 5 , (c) day 10 and (d) at the time of discharge on day 15 
hypo-allergenic, and pain-free. Collagen dressing also provides additional advantage of patients' compliance and comfort. The environment they provide enables the body's reparative and immune systems to function most effectively.

Collagen sheets are very useful in first- and second-degree burns, where they are almost routinely used. The cost of repeated dressings comes down, and the pain associated with such procedures can be avoided. This is especially useful in children.

The biological advantages of collagen sheet can be summarised thus:

- Collagen sheets are non-inflammatory

- They facilitate migration of fibroblasts and microvascular cells

- They help in the synthesis of neodermal collagen matrices

- They have low antigenicity

- They have minimal biodegradation

- They are non-toxic

- They help in minimising scarring

The physiological advantages of the collagen sheets are:

- They are impermeable to bacterial migration

- They modulate fluid flux from the wound

- They are elastic, soft, and supple, and therefore fit all contours

- They have good tear strength

- They have enough strength to be peeled off the wound

Dressing materials like calcium alginate, hydrocolloid membranes and fine mesh gauze have a disadvantage in that they become permeable to bacteria. Biological dressings like collagen, on the other hand, create the most physiological interface between the wound surface and environment, and are impermeable to bacteria.. ${ }^{[1]}$ As compared to conventional dressings, collagen sheet dressings have the advantage of ease of application and are natural, non-immunogenic, non-pyrogenic, hypoallergenic, and pain-free. ${ }^{[2,3]}$ The fact that it makes large areas of ulcerated skin pain free is of particular importance in patients of TEN as ulcers in the mucosal surfaces of buccal cavity, nasal cavity, conjunctiva, urethra, vagina, and anal canal are both painful and bleeding. The use of collagen dressing has been found to inhibit the action of metalloproteinases. ${ }^{[4]}$ Collagen is a biomaterial that encourages wound healing through deposition and organisation of freshly formed fibres and granulation tissue in the wound bed, thus creating a good environment for wound healing. ${ }^{[5]}$ Repeated bleeding from the cutaneous lesions by shearing of the gauze dressings is also effectively countered by collagen sheets. Collagen sheets, when applied to a wound, not only promote angiogenesis, but also enhance body's repair mechanisms. ${ }^{[1,2]}$ While acting as a mechanical support, these reduce oedema and loss of fluids from the wound site, along with facilitation of migration of fibroblasts into the wound and enhancing the metabolic activity of the granulation tissue ${ }^{[1,6,7]}$ Moreover, they are easy to apply and have the additional advantage of stopping bleeding, a property which comes handy in patients of TEN. ${ }^{[8]}$

Other commonly used biological dressings include amniotic membrane and homograft skin. ${ }^{[9]}$ Human amniotic membrane is easy to obtain from elective C-sections, has a low price and provides good wound coverage and has distinct advantages compared with other biologic dressings. ${ }^{[9]}$ Although the risk of transmission of viral infections (e.g. hepatitis, syphilis and HIV) is an important concern with the use of amniotic membrane, with routine screening of each and every patient, this risk can be easily avoided. Thus, for treating burns, many authors have recommended amniotic membrane strongly, but we could not find its mention in the treatment of TEN..$^{[10-13]}$

Additionally, it can be helpful to prevent shear forces and other mechanical disruptions to prevent further areas of skin desquamation. Hence, we feel that collagen sheet dressings are invaluable in treating patients of toxic epidermal necrolysis.

\section{REFERENCES}

1. Park SN, Lee HJ, Lee $\mathrm{KH}$, Suh H. Biological characterization of EDC-crosslinked collagen-hyaluronic acid matrix in dermal tissue restoration. Biomaterials 2003;24:1631-41.

2. Lazovic G, Colic M, Grubor M, Jovanovic M. The application of collagen sheet in open wound healing. Ann Burns Fire Disasters 2005;18:151-6.

3. Horch RE, Stark GB. Comparison of the effect of a collagen dressing and polyurethane dressing on healing of split thickness skin graft donor sites. Scand J Plast Reconst Surg Hand Surg 1998;32:407-13.

4. Veves A, Sheehan P, Pham HT. A randomized, controlled trial of promogran (a collagen/oxidized regenerated cellulose dressing) vs standard treatment in the management of diabetic foot ulcers. Arch Surg. 2002;137:822-7.

5. Nataraj C, Ritter G, Dumas S, Helfer FD, Brunelle J, Sander TW. Extra cellular wound matrices: Novel stabilization and sterilization method for collagen-based biologic wound dressings. Wounds 2007;19:148-56.

6. Nagata $H$, Ueki $H$, Moriguchi T. Fibronectin: Localization in 
normal human skin, granulation tissue, hypertrophic scar, mature scar, progressive systemic sclerotic skin, and other fibrosing dermatoses. Arch Dermatol 1985;121:995-9.

7. Motta G, Ratto GB, De Barbieri A, Corte G, Zardi L, Sacco A, et al. Can heterologous collagen enhance the granulation tissue growth? An experimental study. Ital J Surg Sci 1983;13:101-8.

8. Chung J, Wang XQ, Lindberg FP, Frazier WA. Thrombospondin-1 acts via IAP/CD47 to synergize with collagen in alpha2beta1mediated platelet activation. Blood 1999;94:642-8.

9. Ramakrishnan KM, Jayaraman V. Management of partialthickness burn wounds by amniotic membrane: A cost effective treatment in developing countries. Burns 1997;23:S33-6.

10. Hadjiiski O, Anatassov N. Amniotic membranes for temporary burn coverage. Ann Burns Fire Disasters 1996;9:88-92.
11. Gruss JS, Jirsch DW. Human amniotic membrane: A versatile wound dressing. Can Med Assoc J 1978;118:1237-46.

12. Ghalambor AA, Mohammad HP, Khodadadi A. The amniotic membrane: A suitable biological dressing to prevent infection in thermal burns. Med J Islamic Acad Sci 2000;13:115-8.

13. Mohammadi1 AA, Riazi1 H, Hasheminasab MJ, Sabet $B$, Mohammadi MK, Abbasi S, et al. Amniotic membrane dressing $\mathrm{v} / \mathrm{s}$ conventional topical antibiotic dressing in hospitalized burn patients. Iranian Red Crescent Med J 2009;11:66-70.

How to cite this article: Bhattacharya S, Tripathi HN, Gupta V, Nigam B, Khanna A. Collagen sheet dressings for cutaneous lesions of toxic epidermal necrolysis. Indian J Plast Surg 2011;44:474-7.

Source of Support: Nil, Conflict of Interest: None declared.

Announcement

\section{Android App}

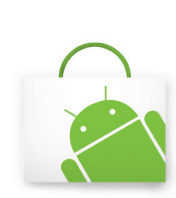

A free application to browse and search the journal's content is now available for Android based mobiles and devices. The application provides "Table of Contents" of the latest issues, which are stored on the device for future offline browsing. Internet connection is required to access the back issues and search facility. The application is compatible with all the versions of Android. The application can be downloaded from https://market.android.com/details?id=comm.app.medknow. For suggestions and comments do write back to us. 